\title{
Risk-Mitigated Optimal Power Flow for Wind Powered Grids
}

\author{
Emma Sjödin, Dennice F. Gayme and Ufuk Topcu
}

\begin{abstract}
Increased penetration of renewable energy sources poses new challenges to the power grid. Grid integrated energy storage combined with fast-ramping conventional generation can help to address challenges associated with power output variability. This paper proposes a risk mitigating optimal power flow (OPF) framework to study the dispatch and placement of energy storage units in a power system with wind generators that are supplemented by fast-ramping conventional back-up generators. This OPF with storage charge/discharge dynamics is solved as a finite-horizon optimal control problem. Chance constraints are used to implement the risk mitigation strategy. The model is applied to case studies based on the IEEE 14 bus benchmark system. First, we study the scheduling of spinning reserves and storage when generation and loads are subject to uncertainties. The framework is then extended to investigate the optimal placement of storage across different network topologies. The results of the case studies quantify the need for storage and reserves as well as suggest a strategy for their scheduling and placement.
\end{abstract}

\section{INTRODUCTION}

There is rapidly growing interest in replacing fossil fuel based power generation with renewable energy sources. Factors driving this interest include increasing fossil fuel prices caused by diminishing supplies [1] along with government mandates [2] and incentives aimed at making the electricity grid "greener." High grid penetrations of solar or wind power pose a number of operational challenges and it is widely accepted that substantial changes to the power grid will be needed for penetration levels above 20\% [3]-[5].

A key strategy to address the challenges posed by the intermittent availability of renewable sources is the use of large-scale grid-integrated energy storage. The role of energy storage in power systems has been extensively investigated. An early simulation study in 1981 [6] confirmed the benefits of quickly dispatchable batteries for peak-shaving and power regulation. More recent investigations have focused on the extent to which grid integrated storage can address the challenges posed by renewable energy sources, e.g., [7], [8]. In systems with renewable energy, storage can be used to minimize spilled energy [4], [5], [9], provide high quality ancillary services and decrease the need for additional transmission capacity [10].

Until now, the power industry has dealt with potential failures using deterministic, worst-case dispatch [4]. This approach, which is generally based an N-1 contingency

E. Sjödin is with the School of Electrical Engineering at the Royal Institute of Technology, SE-100 44 Stockholm, Sweden. emms jo@kth. se

D. F. Gayme is with the Dept. of Mechanical Engineering at Johns Hopkins University, Baltimore, MD, USA, 21218. denniced jhu .edu

U. Topcu is with Control and Dynamical Systems at the California Institute of Technology, Pasadena, CA, USA, 91125. utopculeds. caltech.edu criterion, has thus far also successfully accounted for load variability [11]. However, a deterministic criterion is not suitable for systems with high levels of uncertain power supply. In these cases a worst case approach will lead to unnecessarily large operating reserves, which are not economically or environmentally efficient. Thus, a new grid operating paradigm is needed to handle a large penetration of highly variable power sources such as wind energy [10], [12], [13].

The demand for new operating criteria has led to considerable research activity. For example, the authors of [13] compare the conventionally used $\mathrm{N}-1$ contingency criterion to other operating criteria based on generation and load variability. Probabilistic operating criteria, such as loss-of-load probability (LOLP), were introduced in the market clearing algorithm of [11] and this concept of stochastic security was developed further in [14]. The authors of [4] propose a risklimiting dispatch formulation that limits the LOLP using real-time information about generation and loads. Optimal power flow (OPF) based approaches that include intermittent generation have previously been investigated [15], [16] However, these studies did not include energy storage.

The current work looks at risk mitigated OPF in a new way by analyzing a system with a high penetration of wind power and energy storage. Adding storage charge/discharge dynamics to the risk mitigated OPF leads to a finite-horizon optimal control problem, where risk-limiting (LOLP) constraints account for variability in power generation and loads. Storage systems reduce the requirement of the operating reserves to provide instantaneous power balance. Thus, the objective is to minimize the cost of operating reserves while limiting the LOLP through scheduling operating reserves and energy storage. The reserves are assumed to be fast-ramping conventional generators with some pre-allocated capacity. Such planning of reserves and storage units in the face of uncertainty is of interest to system operators who need to predetermine reserve capacity required for a given time horizon (usually one day). However, the formulation is more general in that it allows any pre-specified operating horizon. The problem can then be solved iteratively in a so-called receding horizon setting whereby the increasingly accurate wind forecasts available closer to the time of dispatch allows one to further minimize the use and pre-scheduling of operating reserves. Such an implementation may be of interest for socalled fast energy markets. Finally, we extend the framework to the complementary problem of how to distribute storage capacity across different power networks. Our placement results provide additional information over previous studies, such as [10], [17], because the risk-limiting OPF captures 


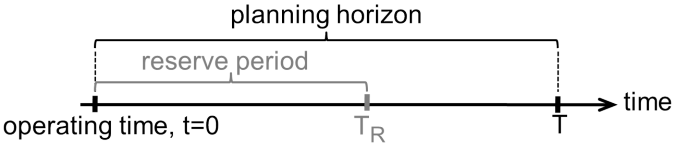

Fig. 1. Illustration of the planning horizon versus the reserve period at time $t=0$. The reserve period defines the time for which reserve capacity is pre-allocated prior to performing the optimization. The finite-horizon OPF is solved over the planning horizon.

system constraints arising from the network topology and Kirchhoff's laws.

The remainder of the paper is organized as follows. The next section describes the problem setup. Section III introduces the risk-limiting OPF with energy storage. Section $\mathrm{V}$ illustrates its use through series of cases studies outlined in Section IV. In Section V-C the framework is extended to study the optimal placement of storage resources using simple network topologies. The paper concludes with a summary and discussion of directions for future work.

\section{Problem SetuP}

Consider a network with a set of buses, $\mathcal{N}$. The set $\mathcal{G} \subset \mathcal{N}$ of generation buses is connected by transmission links to the set $\mathcal{L} \subset \mathcal{N}$ of load buses, i.e., $\mathcal{N}=\mathcal{G} \cup \mathcal{L}$. The generation $g_{k}(t)$ for $k \in \mathcal{G}$ and load (demand) $d_{i}(t)$ for $i \in \mathcal{N}$ are both given and treated as random variables. Fast-ramping conventional generators (spinning reserves) with capacities $S_{k}(t)$ are co-located with the generators at buses $k \in \mathcal{G}$, whereas energy storage units are placed at each bus $i \in \mathcal{N}$.

We define the planning horizon $\mathcal{T}$ with time steps $t \in$ $\mathcal{T}:=\{0,1,2, \ldots, T-1\}$ as the time period over which the optimization takes place. The reserve period $\mathcal{T}_{\mathcal{R}}$, with time steps $t=0, \ldots,\left(T_{R}-1\right)$ and $T_{R} \leq T$, denotes the time for which maximum reserve levels $S_{k}(t)$ at buses $k \in \mathcal{G}$ are pre-determined. The reserve period represents how far in advance operating reserves are scheduled, i.e., when $T_{R}=T$ we assume that the system operator has pre-determined the maximum level of reserves for the entire planning horizon, making $S_{k}(t):=S_{k}$ fixed in time. When $T_{R}<T$ the risk-mitigated OPF determines reserve levels $S_{k}(t)$ for $t=T_{R}, \ldots, T-1$. One may want to have $T_{R}<T$ in a situation where a more accurate wind forecast becomes available $T_{R}$ time steps in advance. In that case, the reserve capacity $S_{k}(t)$ for the time steps $t>T_{R}$ would be updated with the improved estimate. Figure 1 illustrates the planning horizon and the reserve period.

\section{A. Power flows}

The real power flow from bus $i$ to bus $j$ at time $t \in \mathcal{T}$ is

$$
P_{i j}(t)=\left|V_{i}(t)\right|\left|V_{j}(t)\right| B_{i j} \sin \left(\theta_{i}(t)-\theta_{j}(t)\right),
$$

where $i, j \in \mathcal{N} .\left|V_{i}(t)\right|, \theta_{i}(t)$ are respectively the voltage magnitude and angle at bus $i . B_{i j}=B_{j i}$ is the line susceptance between buses $i$ and $j$. The susceptance matrix $B$ models the transmission links in the DC power flow model, which is commonly regarded in dispatch problems.
We adopt this linear DC power flow approximation here. Therefore, we assume that the voltage magnitudes $\left|V_{i}(t)\right|$ for all $t \in \mathcal{T}, i \in \mathcal{N}$ are identically equal to the base voltage $V_{o}=1$ and that the voltage angle differences are small enough that $\sin \left(\theta_{i}(t)-\theta_{j}(t)\right) \approx \theta_{i}(t)-\theta_{j}(t)$ for all $i, j \in \mathcal{N}$. The resulting expression for the power flow is

$$
P_{i j}(t)=B_{i j}\left[\theta_{i}(t)-\theta_{j}(t)\right],
$$

where by abuse of notation we also use $P_{i j}(t)$ to represent the linear approximation. The small angle assumption imposed in (1) can be enforced by requiring

$$
\left|\theta_{i}(t)-\theta_{j}(t)\right| \leq \Theta, \text { for } i, j \in \mathcal{N} \text { and } t \in \mathcal{T} .
$$

Line power capacity limits $\bar{p}_{i j}$ restrict heating of the lines and enforce network stability requirements [18]. These limits bound the power flow between nodes $i$ and $j$ at each $t \in \mathcal{T}$ as

$$
\left|B_{i j}\left(\theta_{i}(t)-\theta_{j}(t)\right)\right| \leq \bar{p}_{i j}, \quad \text { for } i, j \in \mathcal{N} .
$$

As previously discussed, the operating reserves used herein are fast-ramping conventional generators (or spinning reserves) with capacity $S_{k}(t)$ for $k \in \mathcal{G}$. At time $t \in \mathcal{T}$ these reserves are bounded as

$$
0 \leq s_{k}(t) \leq S_{k}(t)
$$

where $S_{k}(t)$ is the maximum amount of reserves available at time $t$. The lower bound in (4) reflects the fact that we assume the reserves to be dispatchable and used in a manner similar to ancillary services in the current power system operating paradigm, i.e. at time $t, s_{k}(t)=0$ unless the available power does not meet the demand (including storage charging requirements).

At each time $t \in \mathcal{T}$, the power flow into the energy storage unit located at bus $i \in \mathcal{N}$ is denoted $r_{i}(t)$, where $r_{i}(t)$ can either be negative (charging) or positive (discharging). This flow in and out of the storage is constrained as

$$
R_{i}^{\min } \leq r_{i}(t) \leq R_{i}^{\max },
$$

where $R_{i}^{\text {min }}<0$. The energy level of the storage at bus $i \in \mathcal{N}$ at $t \in \mathcal{T}$ is then related to the charge/discharge rate through the difference equation

$$
b_{i}(t)=b_{i}(t-1)+r_{i}(t) \Delta t,
$$

where $\Delta t$ is the length of the time step and the initial condition is $b_{i}(0)=b_{0} \geq 0$. The storage level is bounded by each unit's maximum capacity $E_{i}^{\max }$ such that

$$
0 \leq b_{i}(0)+\sum_{\tilde{t}=0}^{t} r_{i}(\tilde{t}) \leq E_{i}^{\max },
$$

for each $i \in \mathcal{N}$ and every $t \in \mathcal{T}$. We also require the energy storage level at the final time to be at least as much as at the beginning of the interval, which is captured by

$$
b_{i}(0) \leq b_{i}(0)+\sum_{\tilde{t}=0}^{T} r_{i}(\tilde{t}) \leq E_{i}^{\max }, \quad \text { for } i \in \mathcal{N} .
$$




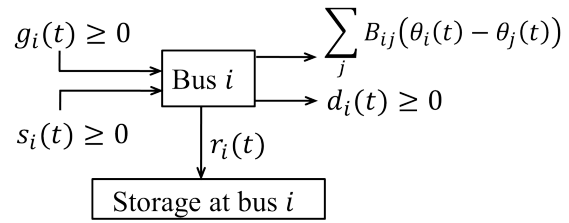

Fig. 2. The in- and out-flow of power at bus $i \in \mathcal{N}$. For $i \in \mathcal{L}, g_{i}(t)=$ and $s_{i}(t)=0$.

\section{B. Risk mitigation strategy}

Under normal operating conditions, the total power in- and out-flows at each bus, shown in Figure 2, must balance, i.e.

$$
g_{i}(t)+s_{i}(t)=d_{i}(t)+r_{i}(t)+\sum_{j \neq i} B_{i j}\left[\theta_{i}(t)-\theta_{j}(t)\right]
$$

for $i \in \mathcal{N}$ and $t \in \mathcal{T}$.

Remark 1: An inequality can also be used in (9) (i.e., $\left.g_{i}(t)+s_{i}(t) \geq d_{i}(t)+r_{i}(t)+\sum_{j \neq i} B_{i j}\left[\theta_{i}(t)-\theta_{j}(t)\right]\right)$ because load balancing is a minimal requirement. As is the current practice, when the wind power availability exceeds demand, excess power can be curtailed.

In a situation with a high penetration of variable renewable energy sources, power balance at each time-step becomes a stochastic problem. The LOLP can then be defined as a risk measure to evaluate compliance with operating conditions. To this end, we define the margin $M_{i}(t)$ between the power in-flow and the power out-flow at bus $i \in \mathcal{N}$ and time $t \in \mathcal{T}$ as

$$
\begin{aligned}
M_{i}(t):= & g_{i}(t)+s_{i}(t) \\
& -\left[d_{i}(t)+r_{i}(t)+\sum_{j \neq i} B_{i j}\left(\theta_{i}(t)-\theta_{j}(t)\right)\right] .
\end{aligned}
$$

As discussed above, $g_{i}(t)$ and $d_{i}(t)$ are given and treated as random variables. We set $g_{i}(t)=0$ if $i \in \mathcal{N} \backslash \mathcal{G}$.

Consider a given scalar $0 \leq \varepsilon_{i} \leq 1$ that defines the accepted risk level, i.e., a bound on the LOLP at bus $i$. We define the following probabilistic operating criterion

$$
\operatorname{Prob}\left\{M_{i}(t) \geq 0\right\} \geq 1-\varepsilon_{i}
$$

for all $i \in \mathcal{N}$ and times $t \in \mathcal{T}$. Constraints such as the one in (10) are so-called chance constraints (see e.g. [19]), which we now reformulate using the theory given in e.g. [20].

Consider the random variable $a$ such that

$$
\text { Prob }\left\{a^{T} x \leq b\right\} \geq \eta
$$

and $\eta \geq 0.5$. If $a$ is normally distributed with mean $\mu$ and covariance $\Sigma$, then Prob $\left\{a^{T} x \leq b\right\}=\Phi\left(\frac{b-\mu^{T} x}{\sqrt{x^{T} \Sigma x}}\right)$, where $\Phi$ is the cumulative distribution function. Now (11) can be rewritten as

$$
b-\mu^{T} x \geq \Phi^{-1}(\eta)\left\|\Sigma^{1 / 2} x\right\|_{2},
$$

where $\eta$ is the confidence level.

We assume that load and wind power estimates, $d_{k}(t)$ and $g_{k}(t)$ in (9), are normally distributed. A normal distribution is a standard assumption for loads [21]. In general, wind power output is a nonlinear function of wind speed, which is assumed to be Rayleigh distributed [22]. Here, as in a number of other studies, e.g. [14], [23], the normal approximation of wind power forecasts is used for mathematical convenience. In addition, the use of this assumption can be attributed to the fact that we are modeling a wind powered grid, which implicitly assumes a large number of geographically dispersed wind farms. The law of large numbers indicates that statistical smoothing effects due to the geographical dispersion of a large number of wind farms may result in the aggregate power approaching a normal distribution, as seen in studies such as [24]. The normality assumption allows for a simple reformulation of the chance constraint of (11). However, the formulation can also be studied with other distributions by introducing additional mathematical machinery such as Chernoff's bounding method, see e.g. [25]. That method allows probability distribution functions from the exponential family to be reformulated into deterministic bounds. However, the results obtained may be more conservative because they can only provide lower bounds on the probabilities, whereas a normal distribution provides an equivalence between (11) and (12).

Let the means and covariances of wind generation and loads be $\bar{g}_{i}(t), \Sigma_{i}^{G}$ and $\bar{d}_{i}(t), \Sigma_{i}^{D}$ respectively and define $\bar{M}_{i}(t):=b-\mu^{T} x$. Then using (11) and (12), the risk-limiting constraint (10) becomes

$$
\bar{M}_{i}(t) \geq \Phi^{-1}\left(1-\varepsilon_{i}\right)\left\|\left(\Sigma_{i}^{G}+\Sigma_{i}^{D}\right)^{1 / 2}\right\|_{2}
$$

for $i \in \mathcal{N}, t \in \mathcal{T}$. Here, the parameters in (12) are defined as

$$
\begin{aligned}
\mu^{T}(t) & :=-\left(\bar{g}_{i}(t)-\bar{d}_{i}(t)\right), \text { and } \\
b & :=-r_{i}(t)+s_{i}(t)+\sum_{j} B_{i j}\left(\theta_{i}(t)-\theta_{j}(t)\right) .
\end{aligned}
$$

\section{RISK-LIMITING OPF}

The goal of the risk-limiting OPF is to schedule the use of fast-ramping generators (reserves) and energy storage units over the finite time horizon $\mathcal{T}$ with minimal cost. Capital costs are not considered in this analysis.

Let the cost of allocating spinning reserve capacity $S_{k}(t)$ for $t$ outside the reserve period (as shown in Figure 1) be given by

$$
c_{R}(S):=\sum_{i \in \mathcal{G}} \sum_{t=T_{R}}^{T-1} H_{i}\left(S_{i}(t), t\right) .
$$

We define cost of using reserve power $s_{k}(t)$ as

$$
c_{s}(s):=\sum_{i \in \mathcal{G}} \sum_{t=0}^{T-1} h_{i}\left(s_{i}(t), t\right)
$$

and denote the cost of storage use by $c_{B}(r)$.

The risk-limiting OPF problem with energy storage is then

$$
\min c_{s}(s)+c_{R}(S)+c_{B}(r)
$$

subject to (2)-(8) and (13) over the decision variables $r_{i}(t), \theta_{i}(t)$ for $i \in \mathcal{N}$ and $t \in \mathcal{T}, s_{k}(t)$ for $k \in \mathcal{G}$ and $t \in \mathcal{T}$, and $S_{k}(t)$ for $k \in \mathcal{G}$ and $t=T_{R}, \ldots, T-1$. 


\section{A. Optimal storage placement}

The risk-mitigated OPF framework can be extended to investigate the optimal distribution of storage throughout the network. This is an important system design question for system planners who need to determine the optimal location to build a storage facility or to evaluate the benefit of siting storage at the loads versus with generation plants.

To study these issues we change the storage capacity $E_{i}^{\max }$ limit at each bus $i \in \mathcal{N}$ in (7) from a given fixed value to a decision variable. We also add a total storage capacity constraint that bounds the sum of these new decision variables as

$$
\sum_{i \in \mathcal{N}} E_{i}^{\max } \leq E^{t o t} .
$$

The storage charge/discharge rate remains bounded as in (5). This optimal storage placement problem then distributes a given amount of storage capacity with given power ratings over all of the available buses under the constraints (3) - (8), (10) and (17) while minimizing the cost function (16).

\section{CAse Studies}

We study the risk-limited OPF problem using the topology of the IEEE 14 bus benchmark system [26], which is representative of a portion of the Midwestern US transmission grid. This section describes the data sources and parameters used for the case studies discussed in Section V. The optimization problem was implemented numerically as a semidefinite program in MATLAB and solved for various cost functions and reserve periods using YALMIP [27].

\section{A. Wind generation data}

The IEEE 14 bus test case contains five generator buses. Generation profiles for these were created using data from five Southern Californian locations provided by the National Renewable Energy Laboratory (NREL) [28]. At each location, the data for five individual wind turbines was averaged at 10 minute intervals for the month of July 2006. The statistics for an average July day were then computed using the 31 days of data. The generation curves were then scaled so that the total energy produced over the 24 hour period matched the total demand, i.e. $\sum_{i \in \mathcal{N}} \sum_{t=0}^{T}\left(g_{i}(t)-d_{i}(t)\right)=$ 0 . This allows us to assume that the installed wind capacity is sufficient to cover the average demand. The top panel of Figure 3 shows the average generation profiles for the 5 generation buses.

\section{B. Load data}

Load profiles were created using normalized demand data from 14 typical Southern California feeders ${ }^{1}$ for the month of July 2010. The data was interpolated to obtain points separated by ten minute intervals to match the wind generation data. Statistics were obtained by averaging over the 31 days of the month for each of the 14 load buses. The demand curves as well as the covariances were then scaled so that the peak values on the demand curves match the loads in the

\footnotetext{
${ }^{1}$ Data was provided by Southern California Edison
}

IEEE 14 bus test case. The bottom panel of Figure 3 shows the resulting demand profiles for each bus.

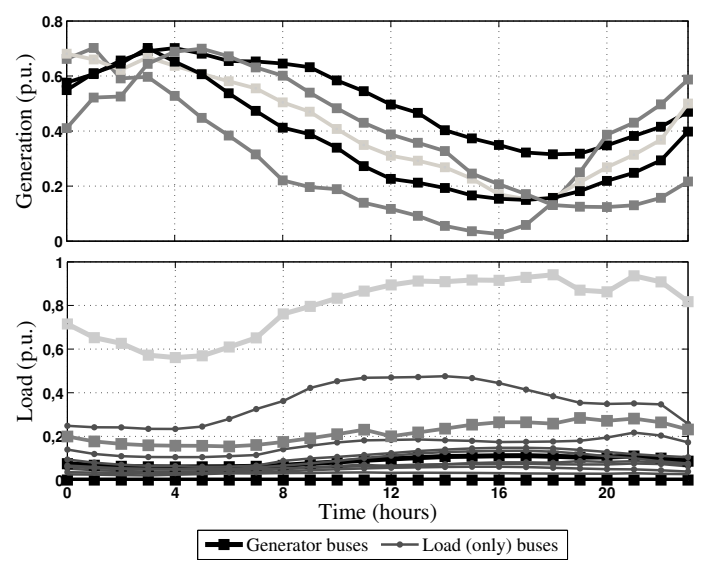

Fig. 3. Generation profiles for the five generator buses based on five Southern Californian wind farms (upper). Demand data for 14 typical feeders (lower). The data represents the generation and demand for an average day in July. These figures are plotted with hourly sub-sampling of the data.

\section{Risk and uncertainty parameters}

The LOLP is limited by the constraint (13). We call the right hand side of (13) for each bus $i \in \mathcal{N}$ the confidence margin because it represents the additional supply (i.e. over the required quantity from the right hand side of (9)) which is needed to produce the desired confidence level $1-\varepsilon_{i}$ in (10). This depends on the assumed risk level $\varepsilon$ and the combined covariance of generation and load. The upper panel of Figure 4 illustrates the multiplicative factor $\Phi^{-1}(1-\varepsilon)$ in (13) as a function of $\varepsilon$.

The lower panel of Figure 4 illustrates the confidence margin's dependence on the variance, where the variance is given as a percentage of the nominal generation (2 p.u., i.e., the total generation). Here, the load variance is kept constant (at $5 \%$ of 2 p.u.) and the risk level is set to $\varepsilon=5 \%$. Although this plot is a simplification of the relation used in the case studies, it demonstrates the relationship between the generation variance and the confidence margin.

The covariances over a one day planning horizon are computed using a full month of statistics. This procedure results in a very large standard deviation, which is partially due to the data's large intra-day fluctuations. In particular, the data includes days when the generators were out of use. Therefore, we reduce the variance in our data using a model based on the one suggested in [29]. Even with this modification, actual wind power forecasts for a given planning horizon are generally more accurate than the data used herein, see e.g. [30]. The curve in the lower panel of Figure 4 shows that this overestimate of the variance means that the results obtained here will be conservative. The use of more accurate wind and load prediction models can improve the risk-limiting OPF based predictions for reserve and storage requirements, especially when used within the receding 
horizon implementation described in the introduction. This is a topic of ongoing research.
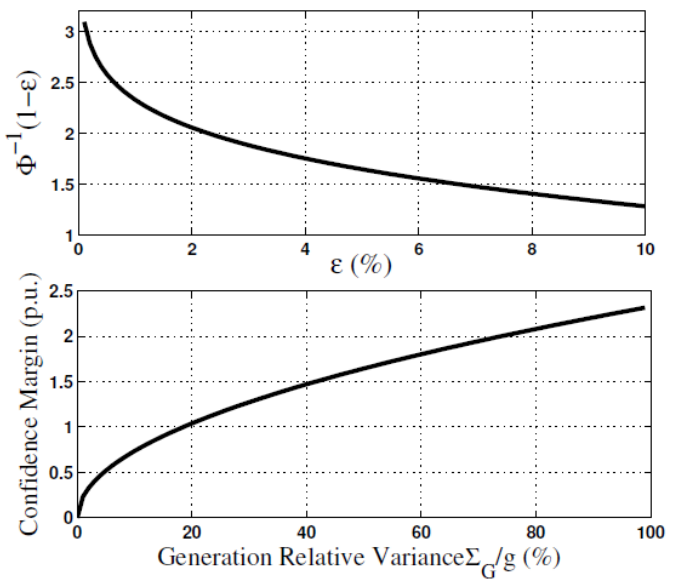

Fig. 4. Upper: the multiplicative factor $\Phi^{-1}(1-\varepsilon)$ from equation (13) as a function of the risk level $\varepsilon$. This function is the inverse cumulative distribution function of the normal probability distribution function. Lower: the confidence margin, i.e. the right hand side of equation (13), as a function of the variance in generation relative to the nominal generation 2 p.u., with load variance $=0.1$ p.u. and $\varepsilon=5 \%$.

\section{Results And Discussion}

In this section we apply the risk-limiting OPF described in Section III to the case studies described in the previous section. We use it to investigate the scheduling of storage and fast-ramping generators using two different reserve periods $\left(T_{R}\right)$. We then apply the modified framework described in Section III-A to determine how the optimal placement and sizing of storage varies with network topology. The risk level and bound on the angle difference in (2) are respectively $\varepsilon_{i}=5 \% \forall i \in \mathcal{N}$ and $\Theta=10^{\circ}$ for all of the examples, unless otherwise indicated. In Examples I and II we assume the storage units have maximum charge (discharge) rates $R_{i}^{\max }=-R_{i}^{\min }=0.5$ p.u. and capacities $E_{i}^{\max }=1.5$ p.u. for all $i \in \mathcal{N}$.

\section{A. Example I}

We first consider the case where spinning reserves are pre-allocated for the entire planning horizon, i.e. the reserve period and planning horizons in Figure 1 are equal, $T_{R}=T$. We fix the spinning reserve limit so that $S_{i}(t)=1$ p.u. for all $i \in \mathcal{G}$ and $t \in \mathcal{T}$. The cost function (16) is made up of quadratic functions $H\left(S_{i}(t)\right)$ and $h\left(s_{i}(t)\right)$, and a linear function $c_{B}\left(\left|r_{i}(t)\right|\right)$, with constant coefficients that are the same at all buses $i \in \mathcal{G}$.

The bottom panel of Figure 5(a) provides a comparison between the total generation-load balance, the confidence margin as well as the fulfilment of the operating criterion (13). Here, it is clear that the generation exceeds the demand until $t=10 \mathrm{~h}$. During this time the storage charges as is seen in the center panel of Figure 5(a). Later in the day, the storage is discharged. The storage is thus combined with spinning reserves to compensate for the generation deficits plus the confidence margin, as depicted in bottom panel of Figure 5(a).

It should be noted that in Example I the full storage capacity is not used. This is because there is never enough surplus energy to both completely charge the storage and compensate for the confidence margin. As expected, the spinning reserves are not used to charge the storage because that would increase the value of the cost function.

\section{B. Example II}

We now consider the case where the reserve period is 6 hours, i.e. $T_{R}=\frac{1}{4} T$, with pre-allocated reserves $S_{i}(t)=1$ p.u. for all $i \in \mathcal{G}$ and $t=0, \ldots, T_{R}-1$. The top and center panels of Figure 5(b) show that this scenario results in the pre-allocated spinning reserves fully charging the storage units during the reserve period. The storage units are then able to minimize the use of reserves later in the day. This behavior is due to the fact that the cost function (14) does not penalize the use of spinning reserves during the reserve period ( $t<T_{R}$ ). It should also be noted that the spinning reserve use in the center panel of Figure $5(\mathrm{~b})$ is piecewise constant due to the quadratic cost function.

The use of reserve capacity to charge energy storage units during off-peak hours is reasonable from a market perspective when low-cost, high efficiency storage is readily available, e.g., in regions where pumped storage is abundant. As mentioned in the introduction, this strategy may also be advantageous from a congestion management perspective depending on the placement of the storage. Storage efficiencies need to be added in order to obtain a complete understanding of the trade-offs between fast-ramping generation based reserves and storage. This is a subject of ongoing research.

\section{Optimal placement problem}

In this section we use simple grid topologies to investigate the optimal placement problem introduced in Section IIIA. Simple topologies allow us to more easily isolate the effects of transmission and network constraints. We expect the insights gained in this simple setting to be applicable to more general network topologies. The three topologies (one 3-node $\Delta$ and two 4-node $\mathrm{Y}$ ) that we investigate are shown in Figure 6. The properties of these networks are again modeled by the susceptance matrix $B$, where a low susceptance implies a long transmission line. Long lines are of particular interest because of the wide separation between generation and load that will result from geographically isolated wind farms being added to the grid [31].

This study uses sinusoidal generation and demand curves with a $\frac{\pi}{2}$ shift between them. There are equal loads at all buses and the generation is scaled so that the total generation aggregated across the time interval ( 24 hours) covers the similarly aggregated total demand. Sinusoids were chosen to approximate the shapes of the previously described data profiles, shown in Figure 3.

For all of the cases tested, any uneven distribution of storage over the network favored a larger share being placed on the generator bus. Therefore, to describe the results in 


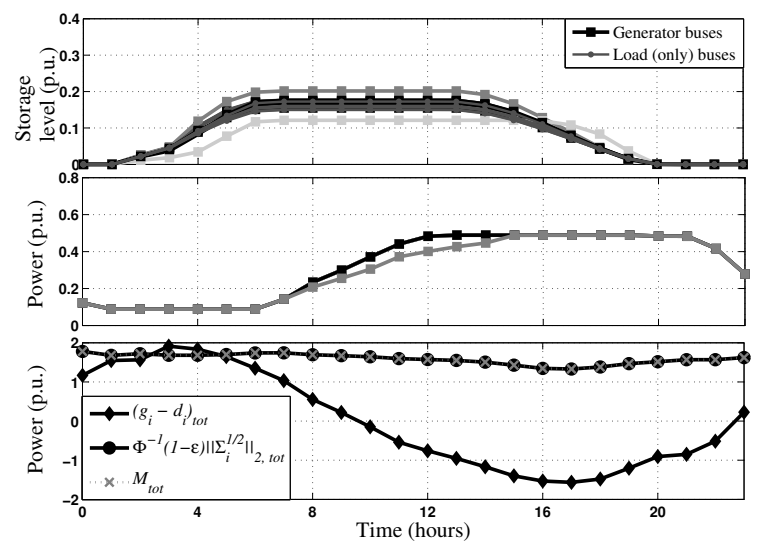

(a)

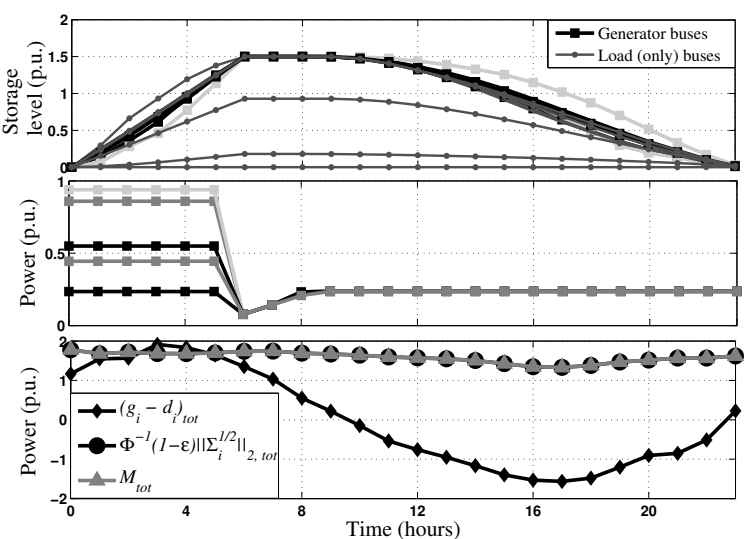

(b)

Fig. 5. Results of optimization program (a) with reserve horizon equal to the planning horizon and (b) with reserve period $T_{R}=6 h$. The figures show storage levels (usage) at each of the 14 buses (top) and spinning reserve usage at the five generator buses (middle) compared to the total generation-load balance and the operating criterion (bottom), where $\left(x_{i}\right)_{\text {tot }}$ denotes $\sum_{i \in \mathcal{N}} x_{i}$.

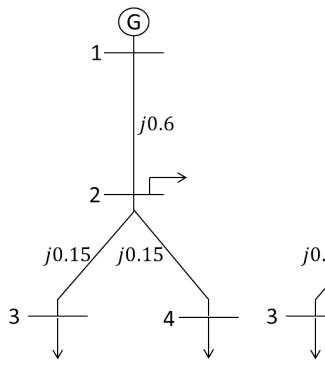

(a)

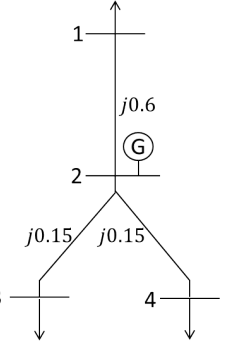

(b)

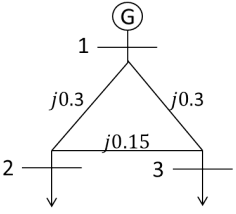

(c)
Fig. 6. Simplified topologies used to study optimal storage placement. (a) and (b) are 4-bus networks with one generator at bus 1 and 2 respectively and three load buses. (c) is a 3-bus topology with one generator at bus 1 and two symmetrically placed loads. The line impedances, in this case purely reactive, are indicated on the lines. The susceptance of each line is the inverse of its reactance.

this section we discuss the percentage by which the generator bus storage capacity exceeds the average capacity at the other buses. Figure 7 depicts this quantity for a number of different test settings. It shows that the advantage gained by placing more storage at the generator bus is largely a function of the power flow line limits (3). This dependence is illustrated by altering the angle bound $\Theta$ in (2). $\Theta=10^{\circ}$ permits a larger power flow and results in more evenly distributed storage than $\Theta=5^{\circ}$, (note that both angles are well in the limits of the small angle assumption). The diagram in Figure 7 also illustrates the strong dependence on the total storage capacity. The topology also plays a role, for example the one in Figure 6(a) favors placing storage at the generator bus more than the other two topologies in Figure 6.

These results can be interpreted as follows. Since the DC power flow approximation models a lossless system, having to transmit power through the network does not have any disadvantages. Therefore when there is enough capacity, an even distribution of the storage is preferable because it can maximize the total power rate delivered by the storage units when the rate is independent of the capacity (as in the current

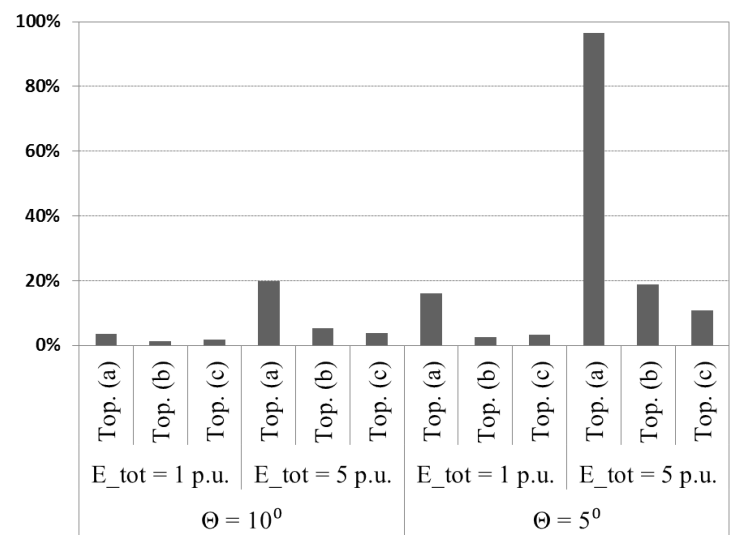

Fig. 7. Result of placement problem for topologies given in Figure 6 given as the percentage with which the storage capacity at the generator node exceeds the mean capacity at the other nodes, with different total capacities and power flow constraints. "Top (a)", "Top (b)" and "Top (c)" refer to the topologies of Figure 6(a)-6(c) respectively.

study). However, when the transmission capacity is limited such that the peak generation can not be transmitted, it is advantageous to store the energy where it is generated. The storage then discharges when there is no congestion. This strategy is increasingly favored as the storage capacity and isolation of the generator bus is increased, as illustrated by the results for topology (a), which has the longest distance to the load buses. When the generator is moved to bus 2 in the same topology (b), power can be transmitted through more than one line and less storage is needed at the generator. For these reasons, buses with very high loads and highly interconnected nodes may also be strategic for storage placement.

\section{Summary AND Potential EXTENSIONS}

We have formulated an optimal power flow problem with risk-limiting constraints to study the scheduling of spinning reserves and energy storage as a finite-horizon optimal control problem. Our formulation models the pre-allocation of reserves for a portion of the planning (optimization) horizon. 
A natural extension of this work would be a receding horizon implementation, where the pre-allocation for some reserve period is determined at each update. Such a framework may be useful in studying different market strategies for scheduling of reserves. For example, day-ahead versus hourahead markets could be compared by changing the reserve period described in Figure 1.

We used this risk-limited OPF to investigate the effects of wind and load variability on the scheduling of spinning reserves and storage units using case studies based on the IEEE 14 bus benchmark system. We modeled an extreme case with wind as the primary energy source and compensate for the variability using reserves that are allocated based on probabilistic operating criteria. The reserve requirements computed in our study represent a worst-case scenario because no wind or load forecasting is modeled. More accurate wind prediction models, time correlations and data forecasting techniques for wind and load data are directions for future work. Data forecasting techniques would be especially beneficial in the receding horizon framework.

The storage placement problem introduced here would benefit from the extension of this framework to an AC power flow setting and the introduction of storage efficiencies. In the lossless DC power flow model discussed herein colocating storage with wind seems to be a preferable strategy. However, the results may differ when transmission losses and voltage drop are included in the analysis. In addition, the use of storage efficiencies would permit cost benefit analysis of different storage technologies and determine whether the type of storage plays a role in placement decisions. Both of these topics are subjects of our ongoing research.

\section{ACKNOWLEDGEMENTS}

The authors acknowledge the support of NSF through grant CNS-0911041, Southern California Edison (SCE) and the Boeing Corporation. A special thank you to Christopher Clarke of SCE for providing data. We are also grateful to Steven Low and K. Mani Chandy of the California Institute of Technology for their insightful comments and interesting discussions.

\section{REFERENCES}

[1] G. Boyle, Ed., Renewable Energy: Power for a Sustainable Future, 2nd ed. Oxford University Press, 2004

[2] U.S. Energy Information Administration, "Annual energy outlooks 2010 with projections to 2035," U.S. Department of Energy, Tech. Rep. DOE/EIA-0383, 2010. [Online]. Available: http://www.eia.doe.gov/oiaf/aeo

[3] F. van Hulle, "Large scale integration of wind energy in the european power supply: analysis, issues and recommendations," EWEA, The European Wind Energy Association, Tech. Rep., 2005.

[4] P. Varaiya, F. Wu, and J. Bialek, "Smart operation of smart grid: Risklimiting dispatch," Proc. of the IEEE, vol. 99, no. 1, pp. 40 -57, Jan. 2011.

[5] Y. M. Atwa and E. F. El-Saadany, "Optimal allocation of ESS in distribution systems with a high penetration of wind energy," IEEE Trans. on Power Systems, vol. 25, no. 4, pp. 1815-1822, Nov. 2010.

[6] T. Yau et al. , "Effects of battery storage devices on power system dispatch," IEEE Trans. on Power Apparatus and Systems, vol. PAS100, no. 1, pp. 375-383, 1981.
[7] M. Geidl and G. Andersson, "A modeling and optimization approach for multiple energy carrier power flow," in Proc. of IEEE PES PowerTech, 2005.

[8] E. Sortomme and M. A. El-Sharkawi, "Optimal power flow for a system of microgrids with controllable loads and battery storage," in Power Systems Conf. and Exposition, 2009.

[9] J. P. Barton and D. G. Infield, "Energy storage and its use with intermittent renewable energy," IEEE Trans. on Energy Conversion, vol. 19, no. 2, pp. 441-448, 2004.

[10] P. Denholm and R. Sioshansi, "The value of compressed air energy storage with wind in transmission-constrained electric power systems," Energy Policy, vol. 37, no. 8, pp. 3149 - 3158, 2009.

[11] F. Bouffard and F. D. Galiana, "An electricity market with a probabilistic spinning reserve criterion," IEEE Trans. on Power Systems, vol. 19, no. 1, pp. $300-307,2004$.

[12] P. Meibom et al., "Stochastic optimization model to study the operational impacts of high wind penetrations in Ireland," IEEE Trans. on Power Systems, vol. 26, no. 3, pp. 1367 -1379, Aug. 2011.

[13] J. Restrepo and F. Galiana, "Effects of wind power on day-ahead reserve schedule," in Proc. of IEEE PES/IAS Conf. on Sustainable Alternative Energy (SAE), Sept. 2009, pp. 1-4.

[14] F. Bouffard and F. D. Galiana, "Stochastic security for operations planning with significant wind power generation," in Proc. of IEEE PES General Meeting, 2008.

[15] R. Jabr, "Optimal Power Flow Using an Extended Conic Quadratic Formulation," IEEE Trans. on Power Systems, vol. 23, no. 3, pp. 10001008, Aug. 2008

[16] H. Ahmadi and H. Ghasemi, "Probabilistic optimal power flow incorporating wind power using point estimate methods," in Proc. of the $10^{\text {th }}$ Int'l Conf. on Env. and Electrical Eng., May 2011, pp. 1 -5.

[17] F. Kienzle and G. Andersson, "Location-dependent valuation of energy hubs with storage in multi-carrier energy systems," in $7^{\text {th }}$ Int'l Conf. on the European Energy Market (EEM), June 2010, pp. 1 -6.

[18] A. R. Bergen and V. Vittal, Power Systems Analysis, 2nd ed. Prentice Hall, 2000.

[19] A. Charnes and W. W. Cooper, "Chance-constrained programming," Management Science, vol. 6, no. 1, pp. 73-79, Oct. 1959.

[20] S. Boyd and L. Vandenberghe, Convex Optimization. Cambridge Univ. Press, 2004.

[21] G. Gross. and F. D. Galiana, "Short-term load forecasting," Proc. of the IEEE, vol. 75, no. 12, pp. 1558 - 1573, 1987.

[22] R. Jabr and B. Pal, "Intermittent wind generation in optimal power flow dispatching," IET Generation, Transmission \& Distribution, vol. 3, no. 1, pp. 66-74, Jan. 2009.

[23] L. Söder, "Reserve margin planning in a wind-hydro-thermal power system," IEEE Trans. on Power Systems, vol. 8, no. 2, pp. $564-571$, May 1993.

[24] U. Focken et al., "Short-term prediction of the aggregated power output of wind farms - a statistical analysis of the reduction of the prediction error by spatial smoothing effects," J. of Wind Engineering \& Industrial Aerodynamics, vol. 90, no. 3, pp. 231 - 246, 2002.

[25] S. Boucheron, G. Lugosi, and O. Bousquet, "Concentration inequalities." [Online]. Available: http://www.econ.upf.edu/ lugosi/mlss_conc.pdf

[26] University of Washington, "Power systems test case archive." [Online]. Available: http://www.ee.washington.edu/research/pstca/

[27] J. Löfberg, "YALMIP : A toolbox for modeling and optimization in MATLAB," in Proc. of the CACSD Conf., Taipei, Taiwan, 2004.

[28] Y. Jiang et al., "Western wind and solar integration study," Tech. Rep., 2005, subcontract report NREL/SR-550-47434. [Online]. Available: http://www.osti.gov/bridge/

[29] A. Fabbri et al., "Assessment of the cost associated with wind generation prediction errors in a liberalized electricity market," IEEE Trans. on Power Systems, vol. 20, no. 3, pp. 1440 - 1446, Aug. 2005.

[30] G. Sideratos and N. Hatziargyriou, "An advanced statistical method for wind power forecasting," IEEE Trans. on Power Systems, vol. 22, no. 1 , pp. $258-265$, Feb. 2007.

[31] A. Mills, R. Wiser, and K. Porter, "The cost of transmission for wind energy: A review of transmission planning studies," Tech. Rep., 2009, US Department of Energy Contract No. DE-AC02-05CH11231. [Online]. Available: http://eetd.lbl.gov/EA/EMP/ 